\title{
Research on The Multi-target Recognition of Millimeter Wave LFMCW Automotive Anti- Collision Radar
}

\author{
Shuang Zhao \\ Changchun University of Science and Technology \\ Chang Chun 130022, China \\ starwarsky@sohu.com
}

\author{
Yunqing Liu \\ Changchun University of Science and Technology \\ Chang Chun 130022, China \\ mzliuyunqing@163.com
}

\begin{abstract}
For linear frequency modulated continuous wave automotive collision avoidance radar system, it has advantages of not existing the blind area, high detection sensitivity, not easy to be interfered and low operating voltage. It becomes the focus of research in recent years. In practice, we often meet the case that there a multi-targets in front of radar. Generally, the radar can distinguish the targets by using stretching and FFT processing. When there are two similar distance and different speeds targets in front of the radar, their one-dimensional FFT spectrum will overlap. In this paper, due to the different speeds of the targets, the speeds can be calculated by the application of the two-dimensional FFT conversion. We use CZT transform to extract the amplitude information of each target. Then the difference between the amplitude of the spectrum is normalized, and the distance of the target is corrected. So the radar can recongize the targets with similar distance but different speed. It greatly enhances the distance measurement accuracy of each target.
\end{abstract}

Keywords-multi-target;LFMCW; anti-collision;millimeter; radar

\section{INTRODUCTION}

Automotive collision avoidance radar can improve the perception of the driver under the poor condition such as rain, fog, dust, night, etc. It can reduce the driver's burden and judgment errors, will strength the road safety and reduce accidents. Automotive collision avoidance radar need to distinguish multiple moving targets. By tracking and classifying the target it provide audible and visible indication [1]. Radar detection of targets is usually under a very complex background [2]. The detection of target, such as a variety of useful information about the angle, moving speed, the distance, shape, and so on is completed by the radar signal processing system. Fast Fourier transform(FFT) is one of the main methods for processing the target echo. It simplified the traditional discrete Fourier transform algorithm, and thus has a higher speed. When it is applied on the radar target echo spectrum processing, it can significantly reduce the processing time and improve the response speed of the radar. Therefore, the FFT analysis becomes the most commonly used method to analyze the signal spectrum of the radar echo.

\section{MULTIPLE TARGETS RECOGNITION OF THE RADAR}

The transmitter waveform in a period of FMCW radar is

$$
x_{1}^{\prime}(t)=x_{1}(t) e^{j \pi \beta t^{2} / t_{0}}
$$

Where $t_{0}$ is the period of the chirp signal, $\beta$ is the bandwidth,

$$
x_{1}(t)=\left\{\begin{array}{lr}
1 & \left(0<t<t_{0}\right) \\
0 & \text { else }
\end{array}\right.
$$

Consider two different targets in front of the radar, which is named target 1 and target 2 . The arriving time of the echoes is respectively $t_{r_{1}}$ and $t_{r_{2}}$. The amplitudes of the echoes are respectively $A_{1}$ and $A_{2}$. After stretching the processed echo signal is as follows:

$$
\begin{aligned}
x_{r}(t)= & A_{1} x_{1}\left(t-t_{r_{1}}\right) \exp \left(j \pi \beta t_{r_{1}}\left(2 t-t_{r_{1}}\right) / t_{0}-j \frac{4 v \pi}{\lambda} t\right. \\
& \left.+2 j \pi f t_{r_{1}}\right)+A_{2} x_{1}\left(t-t_{r_{2}}\right) \exp \left(j \pi \beta t_{r_{2}}\left(2 t-t_{r_{2}}\right) / t_{0}\right. \\
& \left.-j \frac{4 v \pi}{\lambda} t+2 j \pi f t_{r_{2}}\right)
\end{aligned}
$$

Its discrete Fourier transformed spectrum can be written as: 


$$
\begin{aligned}
& x_{r}(k)=A_{1}\left(\frac{\sin \left(C\left(\left(\beta \pi t_{r_{1}} / t_{0}-2 \frac{v \pi}{\lambda}\right) t_{s}-\frac{\pi}{N} k\right)\right)}{\sin \left(\left(\beta \pi t_{r_{1}} / t_{0}-2 \frac{v \pi}{\lambda}\right) t_{s}-\frac{\pi}{N} k\right)}\right) \\
& \times \exp \left(\left(\left(j \beta \pi t_{r_{1}} / t_{0}-2 j \frac{v \pi}{\lambda}\right) t_{s}-j \frac{\pi}{N} k\right)(C-1)\right. \\
& +2 j \pi f t_{r_{1}}+j \pi \beta t_{r_{1}}\left(2\left\lceil\frac{t_{r_{1}}+\Delta t_{2}}{t_{s}}\right\rceil t_{s}-t_{r_{1}}\right) / t_{0} \\
& \left.-j \frac{4 v \pi}{\lambda}\left\lceil\frac{t_{r_{1}}+\Delta t_{2}}{t_{s}}\right\rceil t_{s}-j\left\lceil\frac{t_{r_{1}}+\Delta t_{2}}{t_{s}}\right\rceil \frac{2 \pi}{N} k\right)+ \\
& A_{2}\left(\frac{\sin \left(C\left(\left(\beta \pi t_{r_{2}} / t_{0}-2 \frac{v \pi}{\lambda}\right) t_{s}-\frac{\pi}{N} k\right)\right)}{\sin \left(\left(\beta \pi t_{r_{2}} / t_{0}-2 \frac{v \pi}{\lambda}\right) t_{s}-\frac{\pi}{N} k\right)}\right) \\
& \times \exp \left(\left(\left(j \beta \pi t_{r_{2}} / t_{0}-2 j \frac{v \pi}{\lambda}\right) t_{s}-j \frac{\pi}{N} k\right)(C-1)\right. \\
& +2 j \pi f t_{r_{2}}+j \pi \beta t_{r_{2}}\left(2\left\lceil\frac{t_{r_{2}}+\Delta t_{2}}{t_{s}}\right\rceil t_{s}-t_{r_{2}}\right) / t_{0} \\
& \left.-j \frac{4 v \pi}{\lambda}\left\lceil\frac{t_{r_{2}}+\Delta t_{2}}{t_{s}}\right\rceil t_{s}-j\left\lceil\frac{t_{r_{2}}+\Delta t_{2}}{t_{s}}\right\rceil \frac{2 \pi}{N} k\right)
\end{aligned}
$$

As shown in (3), the spectrum is the addition of two target echo spectrum. The spectrum at point $k_{1}=\left[N\left(\left(\beta t_{r_{1}} / t_{0}-\frac{2 v}{\lambda}\right) t_{s}\right], \quad k_{2}=\left[N\left(\left(\beta t_{r_{2}} / t_{0}-\frac{2 v}{\lambda}\right) t_{s}\right]\right.\right.$ reaches it peak. When the distance between these two spectrums meet the Rayleigh resolution, these two targets can be distinguished. When the distance between these two spectrum does not satisfy the Rayleigh resolution, we do two-dimensional discrete Fourier transform to the sampling point. Let:

$$
\begin{aligned}
x_{r a_{1}}(k)= & A_{1}\left(\frac{\sin \left(C\left(\left(\beta \pi t_{r_{1}} / t_{0}-2 \frac{v \pi}{\lambda}\right) t_{s}-\frac{\pi}{N} k\right)\right)}{\sin \left(\left(\beta \pi t_{r_{1}} / t_{0}-2 \frac{v \pi}{\lambda}\right) t_{s}-\frac{\pi}{N} k\right)}\right) \\
x_{r p_{1}}(k)= & \exp \left(\left(\left(j \beta \pi t_{r_{1}} / t_{0}-2 j \frac{v \pi}{\lambda}\right) t_{s}-j \frac{\pi}{N} k\right)(C-1)\right. \\
& +2 j \pi f t_{r_{1}}+j \pi \beta t_{r_{1}}\left(2\left[\frac{t_{r_{1}}+\Delta t_{2}}{t_{s}}\right\rceil t_{s}-t_{r_{1}}\right) / t_{0} \\
& -j \frac{4 v \pi}{\left.\left.\lambda\left[\frac{t_{r_{1}}+\Delta t_{2}}{t_{s}}\right\rceil t_{s}-j \mid \frac{t_{r_{1}}+\Delta t_{2}}{t_{s}}\right\rceil \frac{2 \pi}{N} k\right)} \\
x_{r a_{2}}(k)= & A_{2}\left(\frac{\sin \left(C\left(\left(\beta \pi t_{r_{2}} / t_{0}-2 \frac{v \pi}{\lambda}\right) t_{s}-\frac{\pi}{N} k\right)\right)}{\sin \left(\left(\beta \pi t_{r_{2}} / t_{0}-2 \frac{v \pi}{\lambda}\right) t_{s}-\frac{\pi}{N} k\right)}\right)
\end{aligned}
$$

$$
\begin{aligned}
x_{r p_{2}}(k)= & \exp \left(\left(\left(j \beta \pi t_{r_{2}} / t_{0}-2 j \frac{v \pi}{\lambda}\right) t_{s}-j \frac{\pi}{N} k\right)(C-1)\right. \\
& +2 j \pi f t_{r_{2}}+j \pi \beta t_{r_{2}}\left(2\left\lceil\frac{t_{r_{2}}+\Delta t_{2}}{t_{s}}\right\rceil t_{s}-t_{r_{2}}\right) / t_{0} \\
& \left.-j \frac{4 v \pi}{\lambda}\left\lceil\frac{t_{r_{2}}+\Delta t_{2}}{t_{s}}\right\rceil t_{s}-j\left\lceil\frac{t_{r_{2}}+\Delta t_{2}}{t_{s}}\right\rceil \frac{2 \pi}{N} k\right)
\end{aligned}
$$

Then

$$
x_{r}(k)=x_{r a_{1}}(k) \times x_{r m_{1}}(k)+x_{r a_{2}}(k) \times x_{r m_{2}}(k)
$$

In $M$ cycles, its spectrum of two-dimensional Fourier transform is:

$$
X_{r}(k)=X_{r a_{1}}(k) \otimes X_{r m_{1}}(k)+X_{r a_{2}}(k) \otimes X_{r m_{2}}(k)
$$

By (9), the spectrum for the two-dimensional Fourier transform is the sum of single target. Thus, when the target speed of the two is greater than the Rayleigh resolution, the same distance, with different speed targets can be distinguished from each other.

\section{DISTANCE REFINEMENT ALGORITHM FOR MULTI- TARGET}

If there are multiple targets which distances are approximate in front of radar , at this time, the spectrums of the targets will overlap. For convenient, we suppose there are two target in front of the radar which distances is similar but at different speeds. When $\beta v M T_{s} / c<<1$, in $\mathrm{M}$ cycles, the echo signal after discrete Fourier transform can be written as: 


$$
\begin{aligned}
& x_{r}(k) \approx A_{1}\left(\frac{\sin \left(C\left(\left(\beta \pi t_{r_{1}} / t_{0}-2 \frac{v \pi}{\lambda}\right) t_{s}-\frac{\pi}{N} k\right)\right)}{\sin \left(\left(\beta \pi t_{r_{1}} / t_{0}-2 \frac{v \pi}{\lambda}\right) t_{s}-\frac{\pi}{N} k\right)}\right) \\
& \times \exp \left(\left(\left(j \beta \pi\left(t_{r_{1}}-2 v n T_{s} / c\right) / t_{0}-2 j \frac{v \pi}{\lambda}\right) t_{s}\right.\right. \\
& \left.-j \frac{\pi}{N} k\right)(C-1)+2 j \pi f\left(t_{r_{1}}-2 v n T_{s} / c\right) \\
& +j \pi \beta t_{r_{1}}\left(2\left[\frac{\left(t_{r_{1}}-2 v n T_{s} / c\right)+\Delta t_{2}}{t_{s}}\right\rceil t_{s}\right. \\
& \left.-\left(t_{r_{1}}-2 v n T_{s} / c\right)\right) / t_{0} \\
& -j \frac{4 v \pi}{\lambda}\left\lceil\frac{\left(t_{r_{1}}-2 v n T_{s} / c\right)+\Delta t_{2}}{t_{s}}\right\rceil t_{s} \\
& \left.-j\left\lceil\frac{\left(t_{r_{1}}-2 v n T_{s} / c\right)+\Delta t_{2}}{t_{s}}\right\rceil \frac{2 \pi}{N} k\right) \\
& +A_{2}\left(\frac{\sin \left(C\left(\left(\beta \pi t_{r_{2}} / t_{0}-2 \frac{v \pi}{\lambda}\right) t_{s}-\frac{\pi}{N} k\right)\right)}{\sin \left(\left(\beta \pi t_{r_{2}} / t_{0}-2 \frac{v \pi}{\lambda}\right) t_{s}-\frac{\pi}{N} k\right)}\right) \\
& \times \exp \left(\left(\left(j \beta \pi\left(t_{r_{2}}-2 v n T_{s} / c\right) / t_{0}-2 j \frac{v \pi}{\lambda}\right) t_{s}\right.\right. \\
& \left.-j \frac{\pi}{N} k\right)(C-1)+2 j \pi f\left(t_{r_{2}}-2 v n T_{s} / c\right)+ \\
& j \pi \beta t_{r_{2}}\left(2\left\lceil\frac{\left(t_{r_{2}}-2 v n T_{s} / c\right)+\Delta t_{2}}{t_{s}}\right\rceil t_{s}\right. \\
& \left.-\left(t_{r_{2}}-2 v n T_{s} / c\right)\right) / t_{0} \\
& -j \frac{4 v \pi}{\lambda}\left\lceil\frac{\left(t_{r_{2}}-2 v n T_{s} / c\right)+\Delta t_{2}}{t_{s}}\right\rceil t_{s} \\
& \left.-j\left\lceil\frac{\left(t_{r_{2}}-2 v n T_{s} / c\right)+\Delta t_{2}}{t_{s}}\right\rceil \frac{2 \pi}{N} k\right)
\end{aligned}
$$

As the two target distance is similar, it is unable to determine the specific location of the target based on the maximum value of the spectrum. To the sampling point $x_{r}(k)$ of $\mathrm{M}$ cycles, doing the discrete Fourier transform, we have:

$$
\begin{aligned}
& X_{r p}(k, l)=A_{1}\left(\frac{\sin \left(C\left(\left(\beta \pi t_{r_{1}} / t_{0}-2 \frac{v \pi}{\lambda}\right) t_{s}-\frac{\pi}{N} k\right)\right)}{\sin \left(\left(\beta \pi t_{r_{1}} / t_{0}-2 \frac{v \pi}{\lambda}\right) t_{s}-\frac{\pi}{N} k\right)}\right) \\
& \times \frac{\sin \left(\frac{\left.\left(\beta \pi v t_{s}(C-1) T_{s}-\beta \pi t_{r_{1}} v T_{s}-2 \pi f t_{0} v T_{s}\right)-c t_{0} \frac{\pi}{M} l\right) M}{c t_{0}}\right)}{(R \pi} \\
& \sin \left(\frac{\left(\beta \pi v t_{s}(C-1) T_{s}-j \beta \pi t_{r_{1}} v T_{s}-c t_{0} \frac{\pi}{M} l-2 \pi f v t_{0} T_{s}\right)}{c t_{0}}\right) \\
& \times \exp \left(\left(\left(j \beta \pi t_{r_{1}} / t_{0}-2 j \frac{v \pi}{\lambda}\right) t_{s}-j \frac{\pi}{N} k\right)(C-1)+2 j \pi f t_{r_{1}}\right. \\
& +\frac{\left(j \beta \pi v t_{s}(C-1) T_{s}-j \beta \pi t_{r_{1}} v T_{s}-j c t_{0} \frac{\pi}{M} l-2 j \pi f v t_{0} T_{s}\right)(M-1)}{c t_{0}} \\
& +j \pi \beta t_{r_{1}}\left(2\left\lceil\frac{t_{r_{1}}+\Delta t_{2}}{t_{s}}\right\rceil t_{s}-t_{r_{1}}\right) / t_{0}-j \frac{4 v \pi}{\lambda}\left\lceil\frac{t_{r_{1}}+\Delta t_{2}}{t_{s}}\right\rceil t_{s} \\
& \left.-j\left[\frac{t_{r_{1}}+\Delta t_{2}}{t_{s}}\right\rceil \frac{2 \pi}{N} k\right)+A_{2}\left(\frac{\sin \left(C\left(\left(\beta \pi t_{r_{2}} / t_{0}-2 \frac{v \pi}{\lambda}\right) t_{s}-\frac{\pi}{N} k\right)\right)}{\sin \left(\left(\beta \pi t_{r_{2}} / t_{0}-2 \frac{v \pi}{\lambda}\right) t_{s}-\frac{\pi}{N} k\right)}\right)
\end{aligned}
$$

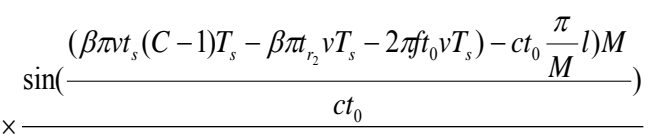

$$
\begin{aligned}
& \sin \left(\frac{\left(\beta \pi v t_{s}(C-1) T_{s}-j \beta \pi t_{r_{2}} v T_{s}-c t_{0} \frac{\pi}{M} l-2 \pi f v t_{0} T_{s}\right)}{c t_{0}}\right) \\
& \times \exp \left(\left(\left(j \beta \pi t_{r_{2}} / t_{0}-2 j \frac{v \pi}{\lambda}\right) t_{s}-j \frac{\pi}{N} k\right)(C-1)+2 j \pi f t_{r_{2}}\right. \\
& +\frac{\left(j \beta \pi v t_{s}(C-1) T_{s}-j \beta \pi t_{r_{2}} v T_{s}-j c t_{0} \frac{\pi}{M} l-2 j \pi f v t_{0} T_{s}\right)(M-1)}{c t_{0}} \\
& +j \pi \beta t_{r_{2}}\left(2\left\lceil\frac{t_{r_{2}}+\Delta t_{2}}{t_{s}}\right\rceil t_{s}-t_{r_{2}}\right) / t_{0}-j \frac{4 v \pi}{\lambda}\left\lceil\frac{t_{r_{2}}+\Delta t_{2}}{t_{s}}\right\rceil t_{s} \\
& \left.-j\left\lceil\frac{t_{r_{2}}+\Delta t_{2}}{t_{s}}\right\rceil \frac{2 \pi}{N} k\right)
\end{aligned}
$$

From (11),we can see that, when the two different target speed satisfy the spectrum resolution of Rayleigh, considering only the effect only by the main lobe of the spectrum, ignoring the side lobes of the spectrum, the two peaks of the spectrum appeared at the point

$$
l_{1}=\left[\frac{M \beta v t_{s}(C-1) T_{s}-\beta t_{r_{1}} v T_{s}-2 f v t_{0} T_{s}}{c t_{0}}\right]
$$

and

$$
l_{2}=\left[\frac{M \beta v t_{s}(C-1) T_{s}-\beta t_{r_{2}} v T_{s}-2 f v t_{0} T_{s}}{c t_{0}}\right],
$$

the amplitude of the two peaks are 


$$
\begin{aligned}
& \left|X_{r p}(k, l)\right|_{\max 1}=A_{1}\left(\frac{\sin \left(C\left(\left(\beta \pi t_{r_{1}} / t_{0}-2 \frac{v \pi}{\lambda}\right) t_{s}-\frac{\pi}{N} k\right)\right)}{\sin \left(\left(\beta \pi t_{r_{1}} / t_{0}-2 \frac{v \pi}{\lambda}\right) t_{s}-\frac{\pi}{N} k\right)}\right) \times \\
& \frac{\sin \left(\frac{\left.\left(\beta \pi v t_{s}(C-1) T_{s}-\beta \pi t_{r_{1}} v T_{s}-2 \pi f t_{0} v T_{s}\right)-c t_{0} \frac{\pi}{M} l\right) M}{c t_{0}}\right)}{(R m} \\
& \sin \left(\frac{\left(\beta \pi v t_{s}(C-1) T_{s}-j \beta \pi t_{r_{1}} v T_{s}-c t_{0} \frac{\pi}{M} l-2 \pi f v t_{0} T_{s}\right)}{c t_{0}}\right) \\
& \left|X_{r p}(k, l)\right|_{\max 2}=A_{2}\left(\frac{\sin \left(C\left(\left(\beta \pi t_{r_{2}} / t_{0}-2 \frac{v \pi}{\lambda}\right) t_{s}-\frac{\pi}{N} k\right)\right)}{\sin \left(\left(\beta \pi t_{r_{2}} / t_{0}-2 \frac{v \pi}{\lambda}\right) t_{s}-\frac{\pi}{N} k\right)}\right) \\
& \times \frac{\sin \left(\frac{\left.\left(\beta \pi v t_{s}(C-1) T_{s}-\beta \pi t_{r_{2}} v T_{s}-2 \pi f t_{0} v T_{s}\right)-c t_{0} \frac{\pi}{M} l\right) M}{c t_{0}}\right)}{\sin \left(\frac{\left(\beta \pi v t_{s}(C-1) T_{s}-j \beta \pi t_{r_{2}} v T_{s}-c t_{0} \frac{\pi}{M} l-2 \pi f v t_{0} T_{s}\right)}{c t_{0}}\right)}
\end{aligned}
$$

From (12) and (13), it can be seen when using the CZT transform to refine the spectrum, two peaks of the spectrum will appear. The amplitudes of the two peaks is

$$
\begin{aligned}
& \left|X_{r p}(k, l)\right|_{\max 1}=A_{1}\left(\frac{\sin \left(C\left(\left(\beta \pi t_{r_{1}} / t_{0}-2 \frac{v \pi}{\lambda}\right) t_{s}-\frac{\pi}{N} k\right)\right)}{\sin \left(\left(\beta \pi t_{r_{1}} / t_{0}-2 \frac{v \pi}{\lambda}\right) t_{s}-\frac{\pi}{N} k\right)}\right) \\
& \left|X_{r p}(k, l)\right|_{\max 2}=A_{2}\left(\frac{\sin \left(C\left(\left(\beta \pi t_{r_{2}} / t_{0}-2 \frac{v \pi}{\lambda}\right) t_{s}-\frac{\pi}{N} k\right)\right)}{\sin \left(\left(\beta \pi t_{r_{2}} / t_{0}-2 \frac{v \pi}{\lambda}\right) t_{s}-\frac{\pi}{N} k\right)}\right)
\end{aligned}
$$

The amplitude of the spectrum represented by (14) and (15) is just the distance spectrum amplitude of target 1 and target 2 .

$$
\begin{aligned}
& \left|X_{r p}(k, l)\right|_{\max 1}=A_{1}\left(\frac{\sin \left(C\left(\left(\beta \pi t_{r_{1}} / t_{0}+2 \frac{v \pi}{\lambda}\right) t_{s}-\frac{\pi}{N} k\right)\right)}{\sin \left(\left(\beta \pi t_{r_{1}} / t_{0}+2 \frac{v \pi}{\lambda}\right) t_{s}-\frac{\pi}{N} k\right)}\right) \\
& \left|X_{r p}(k, l)\right|_{\max 2}=A_{2}\left(\frac{\sin \left(C\left(\left(\beta \pi t_{r_{2}} / t_{0}+2 \frac{v \pi}{\lambda}\right) t_{s}-\frac{\pi}{N} k\right)\right)}{\sin \left(\left(\beta \pi t_{r_{2}} / t_{0}+2 \frac{v \pi}{\lambda}\right) t_{s}-\frac{\pi}{N} k\right)}\right)
\end{aligned}
$$

By (14), (15), (16), (17), we obtain the one-dimensional Fourier transform amplitude of each target. Thus, the distance informations of the multiple targets can be accurately distinguished.

\section{Simulation OF THE ALGORITHM}

Assume there are three targets in front of the radar, the distance frequency of target 1 echo after stretching process is $1 \mathrm{MHz}$, the Doppler frequency shift is $5 \mathrm{kHz}$; the distance frequency of target 2 echo after stretching process is $6 \mathrm{MHz}$, the Doppler frequency shift is $5 \mathrm{kHz}$; the distance frequency of target 3 echo after stretching process is $6 \mathrm{MHz}$, the Doppler frequency shift is $10 \mathrm{kHz}$. So the received signal after strething process is:

$$
\begin{aligned}
x(t)= & 0.5 \sin \left(2 \times 10^{6} \pi t+1 \times 10^{4} \pi t+\varphi_{1}\right) \\
& +0.2 \sin \left(12 \times 10^{6} \pi t+1 \times 10^{4} \pi t+\varphi_{2}\right) \\
& +0.3 \sin \left(12 \times 10^{6} \pi t+2 \times 10^{4} \pi t+\varphi_{3}\right)
\end{aligned}
$$

Set the sampling frequency is $40 \mathrm{MHz}$, do 1024 points FFT. The simulation results can be obtained:

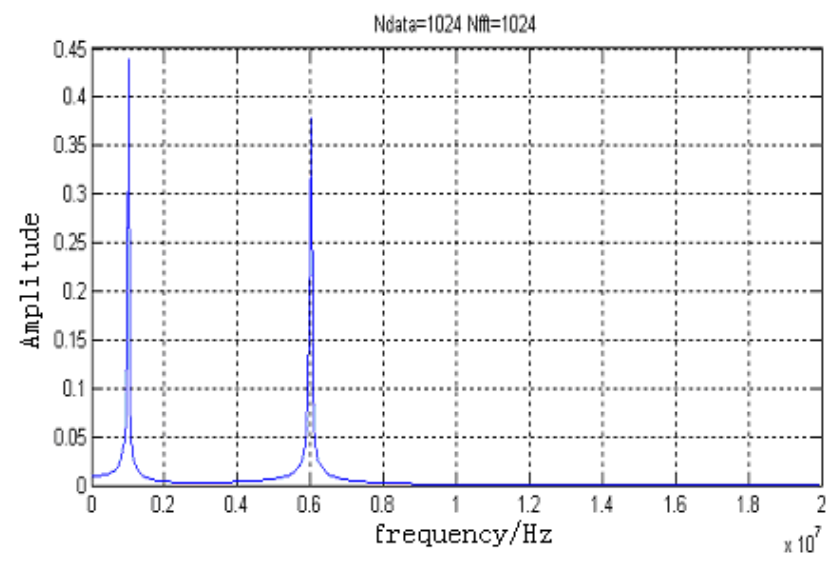

Figure 1. The simulation result of one dimensional FFT

As shown above, we can get the distance information of the target by using one-dimensional FFT. But for the targets which distances are similar, we can not distinguish the target by usingone-dimensional FFT. After doing the two-dimensional FFT, the simulation results is

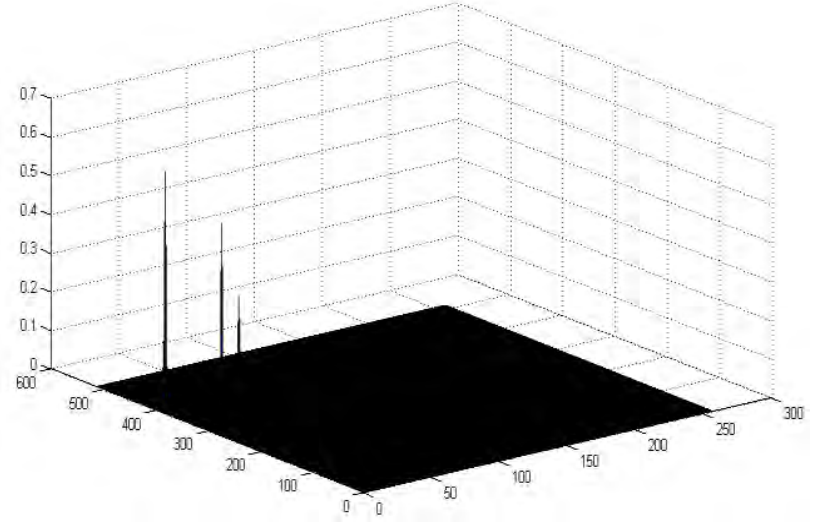

Figure 2. The simulation result of two dimensional FFT

\section{CONCLUSION}

In the applications of the millimeter-wave automotive anti-collision radar, it often encounters the case that multiple targets are in front of the radar. When the two targets have the similar distance but different speed, after one-dimensional FFT, the echo spectrum of the two targets 
will overlap. Thus, the spectral amplitude information of the target can not be extracted. However, due to the different speed of the two targets, we can use CZT transform to refine the speed spectrum, then accurately extract the spectrum amplitude of each target. By using this algorithm, the radar can measure the distance and speed of the target accurately. a substantial increase in the target resolution radar. It significantly improves the ability of radar to distinguish the target.

\section{REFERENCES}

[1] Dai Zhengjian, Qu Cuiping. The resolving method of radar nonrigid multi-target. Radar,2001 CIE International Conference on,Proceedings, 15-18 Oct.2001:1055-1058.

[2] YangJianyu, HuangShunji. Moving target indication of LFMCW radar for clutter rejection. Int.Sym.On Noise and Clutter Rejection in Radars and Imaging Sensors, 1989:585-589.

[3] G.S.Gill, Jen-ChihHuang. The Ambiguity Function of the Step Frequency Radar Signal Processor. Radar, 1996.Proceedings.CIE International Conference of, 8-10 Oct.1996, Pages:375-380.
[4] B-E.Tullsson.Topics in FMCW Radar Disturbance Suppression.Proc.of Radar 97,1997:1-5.

[5] Stove AG.Linear FMCW Radar Techniques. IEE proceedings F.1992(5):343-350.

[6] M. A. Richards, Fundamentals of Radar Signal Processing. McGraw-Hill, NewYork, 2005.

[7] Y. Luo, Q. Zhang, C. W. Qiu, X, J. Liang, and K. M. Li. "Microdoppler effectanalysis and feature extraction in ISAR imaging with stepped-frequency chirp signals" , IEEE Trans. Geosci. Remote Sens., vol. 48, no. 4, pp. 2087-2098, Apr. 2010.

[8] S.D.Howard, A.R.Calderbank.Rwlationships Between Radar Ambiguity and Coding Theory.IEEE International Radar Conference, 2005.898 900.

[9] T. Wang, X. Wang, Y. Chang, J. Liu and S. Xiao, "Estimation of PrecessionParameters and Generation of ISAR Images of Ballistic Missile Targets", IEEE Trans.Aerosp. Electron. Syst" vol. 46, no.4, pp. 1983-1995, Oct. 2010.

[10] X. Y. Pan, W. Wang, J. Liu et al, "Features extraction of rotationally symmetric ballistic targets based on micro-Doppler". PIER, vol. 137, pp. 727-740, 2013. 